\title{
Adaptive Fuzzy Sliding-Mode Control of Uncertain Nonlinear System
}

\author{
Wenda Zheng *, Gang Wu, Gang Xu and Xiangchun Chen \\ Measurement \& Control Technology Research Office, Hefei New Star Institute \\ People's Republic of China \\ wdsubmit@qq.com
}

Keywords: Adaptive Control; Sliding-mode Control; Nonlinear System; Uncertain System; Fuzzy Control

\begin{abstract}
This paper presents an adaptive sliding-mode control algorithm for uncertain nonlinear system. It is very difficult to obtain the exact knowledge and it is required to approximate an unmolded dynamics with a nonlinear component. Therefore, a fuzzy basis function network is applied to approximate the unknown dynamics of nonlinear system. The paper employs a weight factor to adjust the ratio of direct and indirect adaptive fuzzy control, meantime a supervisory controller is introduced and a sliding-mode controller is to ensure optimal tracking performance of the closed-loop system. To prevent the system state variables unpredictable, this paper designs an observer to estimate the unpredictable states. The control structure and learning rules are derived from a Lyapunov theory extension that guarantees both tracking errors and parameter estimate errors in the closed-loop system are bounded. A two-arm robot is simulated to verify the feasibility of the proposed control scheme.
\end{abstract}

\section{Introduction}

The purpose of robot arm control is to maintain the dynamic response of the manipulator in accordance with some pre-specified performance[1]. Although the control problem can be stated in such a simple manner, its solution is complicated for the robot's highly nonlinear dynamics. In general, the control problem consists of obtaining dynamic models of the robotic system and using these models to determine control laws or strategies to achieve the desired system response and performance. Although researchers have proposed many methods, such as the feedback linearization of nonlinear systems, which cancels the nonlinearities of robot manipulators and imposes a desired linear model so that linear control techniques can be applied. However, the method is based on the exact knowledge of robot dynamics. Actually, it is very difficult to obtain the exact knowledge and it is required to approximate an unmodled dynamics with a nonlinear component. Neural networks and fuzzy systems provide good solutions to this challenging task. In this paper, we design an adaptive fuzzy sliding-mode controller for robot manipulators[2-4].

It has been proved that fuzzy basis function (FBF) networks can be universal approximators with arbitrarily small errors [5-7]. Therefore, a fuzzy basis function network is used to approximate and cancel the unknown dynamics of robot manipulators. As in, the control structure and learning rules are derived from a Lyapunov theory extension that guarantee both tracking errors and parameter estimate errors in the closed-loop system are bounded. By taking the uncertainties including approximation errors and external disturbances into consideration, such a technique can reject the effects.

\section{Nonlinear System}

Consider the following nth order nonlinear MIMO system[8], described by: 


$$
\begin{gathered}
y_{1}{ }^{\left(r_{1}\right)}=f_{1}(\boldsymbol{x})+\sum_{j=1}^{n} g_{1 j}(\boldsymbol{x}) u_{j}+d_{1} \\
y_{2}{ }^{\left(r_{2}\right)}=f_{2}(\boldsymbol{x})+\sum_{j=1}^{n} g_{2 j}(\boldsymbol{x}) u_{j}+d_{2} \\
\vdots \\
y_{n}{ }^{\left(r_{n}\right)}=f_{n}(\boldsymbol{x})+\sum_{j=1}^{n} g_{n j}(\boldsymbol{x}) u_{j}+d_{n}
\end{gathered}
$$

where $\boldsymbol{x}=\left[\begin{array}{llllllll}y_{1} & \dot{y}_{1} & \cdots & y_{1}^{\left(r_{1}-1\right)} & \cdots & y_{n} & \cdots & y_{n}^{\left(r_{n}-1\right)}\end{array}\right]^{\mathrm{T}} \in \square^{r}$ is the measurable state vector of the system ; $\boldsymbol{u}=\left[\begin{array}{lll}u_{1} & \cdots & u_{n}\end{array}\right]^{\mathrm{T}} \in \square^{n}$ and $\boldsymbol{y}=\left[\begin{array}{lll}y_{1} & \cdots & y_{n}\end{array}\right]^{\mathrm{T}} \in \square^{n}$ are the input and output of the system; $f_{i}(\boldsymbol{x})$ and $g_{i j}(\boldsymbol{x})$ are unknown continuous functions; $\boldsymbol{d}=\left[\begin{array}{llll}d_{1} & d_{2} & \cdots & d_{n}\end{array}\right]^{\mathrm{T}}$ is the unknown external disturbance, and $d_{i}$ is assumed have the upper bound, i.e. $\left|d_{i}(t)\right| \leq D_{i}, i, j=1,2, \cdots, n, r=r_{1}+r_{2}+\cdots+r_{n}$.

And the Eq. (1) can be rewritten as:

$$
\begin{aligned}
& \boldsymbol{y}^{(r)}=\boldsymbol{F}(\boldsymbol{x})+\boldsymbol{G}(\boldsymbol{x}) \boldsymbol{u}+\boldsymbol{d} \\
& \text { where } \quad \boldsymbol{F}(\boldsymbol{x})=\left[\begin{array}{lllll}
f_{1}(\boldsymbol{x}) & f_{2}(\boldsymbol{x}) & \cdots & f_{n}(\boldsymbol{x})
\end{array}\right]^{\mathrm{T}}, \quad \boldsymbol{G}(\boldsymbol{x})=\left[\begin{array}{lllll}
\boldsymbol{g}_{1}(\boldsymbol{x}) & \boldsymbol{g}_{2}(\boldsymbol{x}) & \cdots & \boldsymbol{g}_{n}(\boldsymbol{x})
\end{array}\right]^{\mathrm{T}}, \quad \boldsymbol{g}_{i}(\boldsymbol{x})=\left[\begin{array}{lll}
\boldsymbol{g}_{i 1}(\boldsymbol{x}) & \cdots & \boldsymbol{g}_{i n}(\boldsymbol{x})
\end{array}\right], \\
& i=1,2, \cdots, n
\end{aligned}
$$

The tracking error is defined as:

$$
\begin{gathered}
e_{1}(t)=y_{d_{1}}(t)-y_{1}(t) \\
\vdots \\
e_{n}(t)=y_{d_{n}}(t)-y_{n}(t)
\end{gathered}
$$

where $\quad \boldsymbol{e}=\left[\begin{array}{llll}e_{1} & e_{2} & \cdots & e_{n}\end{array}\right]^{\mathrm{T}} \quad, \quad \boldsymbol{y}_{d}=\left[\begin{array}{lllll}y_{d_{1}} & y_{d_{2}} & \cdots & y_{d_{n}}\end{array}\right]^{\mathrm{T}} \quad, \quad \boldsymbol{y}_{d}^{(r)}=\left[\begin{array}{llll}y_{d_{1}}^{\left(r_{1}\right)} & y_{d_{2}}^{\left(r_{2}\right)} & \cdots & y_{d_{n}}^{\left(r_{n}\right)}\end{array}\right]^{\mathrm{T}} \quad$, $\boldsymbol{Y}_{d}=\left[\begin{array}{llllllllll}y_{d_{1}} & \dot{y}_{d_{1}} & \cdots & y_{d_{1}}^{\left(r_{1}\right)} & \cdots & y_{d_{n}} & \dot{y}_{d_{n}} & \cdots & y_{d_{n}}^{\left(r_{n}\right)}\end{array}\right]^{\mathrm{T}}, \quad \underline{\boldsymbol{e}}=\boldsymbol{Y}_{d}-\boldsymbol{x}=\left[\begin{array}{lllllllllll}e_{1} & \dot{e}_{1} & \cdots & e_{1}^{\left(r_{1}\right)} & \cdots & e_{n} & \dot{e}_{n} & \cdots & e_{n}^{\left(r_{n}\right)}\end{array}\right]^{\mathrm{T}}, \quad \underline{\boldsymbol{e}}_{i}=\left[\begin{array}{llllll}e_{i} & \dot{e}_{i} & \cdots & e_{i}^{\left(r_{i}\right)}\end{array}\right]^{\mathrm{T}}$, $\underline{\hat{\boldsymbol{e}}}=\boldsymbol{Y}_{d}-\hat{\boldsymbol{x}}=\left[\begin{array}{lllllll}\hat{e}_{1} & \hat{\dot{e}}_{1} & \cdots & \hat{e}_{1}^{\left(r_{1}\right)} & \cdots & \hat{e}_{n} & \hat{\dot{e}}_{n} \cdots \hat{e}_{n}^{\left(r_{n}\right)}\end{array}\right]^{\mathrm{T}}, \quad i=1,2, \cdots, n$.

$\dot{\boldsymbol{Y}}_{d}=\boldsymbol{A} \boldsymbol{Y}_{d}+\boldsymbol{B} \boldsymbol{y}_{d}^{(r)}$

If $\boldsymbol{F}(\boldsymbol{x})$ and $\boldsymbol{G}(\boldsymbol{x})$ are known and free of external disturbance, i.e., $\boldsymbol{d}=\boldsymbol{0}$, we get an ideal control law:

$$
\boldsymbol{u}^{*}=\boldsymbol{G}^{-1}(\boldsymbol{x})\left[-\boldsymbol{F}(\boldsymbol{x})+\boldsymbol{y}_{d}^{(r)}+\boldsymbol{C}_{e}^{\mathrm{T}} \underline{\boldsymbol{e}}\right]
$$

$$
\text { where } \boldsymbol{C}_{e}=\operatorname{diag}\left[\boldsymbol{C}_{e r_{1}} \boldsymbol{C}_{e r_{2}} \cdots \boldsymbol{C}_{e_{n}}\right], \boldsymbol{C}_{e_{r_{i}}}=\left[\begin{array}{llll}
c_{r_{i}}^{e} & c_{r_{i-1}}^{e} & \cdots & c_{1}^{e}
\end{array}\right]^{\mathrm{T}} \text { are the feedback gain matrixes. }
$$

Applying Eq.(5) into Eq.(2):

$$
\underline{\boldsymbol{e}}^{(r)}+c_{r}^{e} \underline{\boldsymbol{e}}^{(r-1)}+\cdots+c_{1}^{e} \underline{\boldsymbol{e}}=0
$$

i.e.

$$
\lim _{t \rightarrow \infty}\|\underline{\boldsymbol{e}}(t)\|=0
$$

However, $\boldsymbol{F}(\boldsymbol{x})$ and $\boldsymbol{G}(\boldsymbol{x})$ can't be exactly known and the disturbance $\boldsymbol{d}$ is uncertain in the real systems.

\section{Algorithm Structure}

It has been proved that FBFN can be universal approximators with arbitrarily small errors [3, 9], assuming that:

$$
\begin{aligned}
& \boldsymbol{F}\left(\hat{\boldsymbol{x}} \mid \Theta_{1}\right)=\boldsymbol{\Phi}(\hat{\boldsymbol{x}}) \Theta_{1} \\
& \boldsymbol{G}\left(\hat{\boldsymbol{x}} \mid \Theta_{2}\right)=\boldsymbol{\Phi}(\hat{\boldsymbol{x}}) \Theta_{2} \\
& \boldsymbol{u}_{d}\left(\hat{\boldsymbol{x}} \mid \Theta_{3}\right)=\boldsymbol{\Phi}(\hat{\boldsymbol{x}}) \Theta_{3}
\end{aligned}
$$

where $\quad \boldsymbol{\Phi}(\hat{\boldsymbol{x}})=\operatorname{diag}\left[\xi^{\mathrm{T}}(\hat{\boldsymbol{x}}) \cdots \xi^{\mathrm{T}}(\hat{\boldsymbol{x}})\right], \quad \Theta_{1}=\left[\begin{array}{lll}\Theta_{11} & \cdots & \Theta_{1 n}\end{array}\right]^{\mathrm{T}}, \quad \Theta_{1 i}=\left[\begin{array}{lll}\theta_{1 i}^{1} & \cdots & \theta_{n i}^{1}\end{array}\right]^{\mathrm{T}}, \quad \Theta_{2}=\left[\begin{array}{lll}\Theta_{21} & \cdots & \Theta_{2 n}\end{array}\right]^{\mathrm{T}}$, $\Theta_{2 i}=\operatorname{diag}\left[\Theta_{1 i}^{2} \cdots \Theta_{n i}^{2}\right]^{\mathrm{T}}, \Theta_{j i}^{2}=\left[\begin{array}{lll}\theta_{1 j i}^{2} & \cdots & \theta_{n j i}^{2}\end{array}\right]^{\mathrm{T}}, \Theta_{3}=\left[\begin{array}{llll}\Theta_{31} & \cdots & \Theta_{3 n}\end{array}\right]^{\mathrm{T}}, \Theta_{3 i}=\left[\begin{array}{lll}\theta_{1 i}^{3} & \cdots & \theta_{n i}^{3}\end{array}\right]^{\mathrm{T}}$.

Choosing the following control law $\boldsymbol{u}$ : 
$\boldsymbol{u}=\boldsymbol{u}_{b}+\boldsymbol{u}_{c}+\boldsymbol{u}_{r}+\boldsymbol{u}_{s}$

where

$\boldsymbol{u}_{b}=\frac{1}{\sigma_{0}} \boldsymbol{L}(s) \boldsymbol{C}_{o}^{\mathrm{T}} \boldsymbol{P}_{2} \underline{\hat{\boldsymbol{e}}}$

$\boldsymbol{u}_{c}=\alpha \boldsymbol{u}_{I}+(1-\alpha) \boldsymbol{u}_{d}\left(\hat{\boldsymbol{x}} \mid \Theta_{3}\right)$

$\boldsymbol{u}_{I}=\hat{\boldsymbol{G}}^{-1}\left(\hat{\boldsymbol{x}} \mid \Theta_{2}\right)\left[-\hat{\boldsymbol{F}}\left(\hat{\boldsymbol{x}} \mid \Theta_{1}\right)+\boldsymbol{y}_{d}^{(r)}+\boldsymbol{C}_{e}^{\mathrm{T}} \underline{\boldsymbol{e}}\right]$

$\boldsymbol{u}_{r}=\frac{1}{2 \sigma_{0}} \boldsymbol{L}(s) \boldsymbol{R}^{-1} \tilde{\boldsymbol{E}}_{1}$

where $\boldsymbol{u}_{b}$ is the feedback controller, and $\boldsymbol{u}_{r}$ is $H_{\infty}$ robust controller, and $\boldsymbol{u}_{s}$ is a supervised controller , and $\boldsymbol{L}(s), \boldsymbol{R}, \tilde{\boldsymbol{E}}_{1}, \boldsymbol{P}_{2}$ are given in the following texts[10].

And the definitions $\boldsymbol{L}(s)=\operatorname{diag}\left[\boldsymbol{L}_{1}(s) \cdots \boldsymbol{L}_{n}(s)\right], \boldsymbol{L}_{i}(s)=s^{m_{i}}+b_{i 1} s^{m_{i}-1}+\cdots+b_{m_{i}}, m_{i}=r_{i}-1, i=1,2, \cdots, n$, which satisfies the following equation:

$\tilde{\boldsymbol{E}}_{1}=\boldsymbol{H}(s) \boldsymbol{L}(s)\left\{\alpha\left[\boldsymbol{L}^{-1}(s)(\hat{\boldsymbol{F}}-\boldsymbol{F})+\boldsymbol{L}^{-1}(s)(\hat{\boldsymbol{G}}-\boldsymbol{G}) \boldsymbol{u}_{\boldsymbol{I}}\right]+(1-\alpha) \boldsymbol{G} \boldsymbol{L}^{-1}(s)\left(\boldsymbol{u}^{*}-\hat{\boldsymbol{u}}_{d}\right)-\boldsymbol{G} \boldsymbol{L}^{-1}(s)\left(\boldsymbol{u}_{b}+\boldsymbol{u}_{r}+\boldsymbol{u}_{s}\right)-\boldsymbol{L}^{-1}(s) \boldsymbol{d}\right\}$

$\boldsymbol{u}_{s}$ is a supervised controller:

$$
\begin{aligned}
\boldsymbol{u}_{s}= & \frac{\boldsymbol{L}(s) I^{*} \operatorname{sgn}\left(\tilde{\boldsymbol{E}}_{1}\right)\left\|\boldsymbol{L}^{-1}\right\|}{\sigma_{0}}\left[\alpha \sigma_{0}\left\|\boldsymbol{u}_{I}\right\|+(1-\alpha) \sigma_{0}\left\|\hat{\boldsymbol{u}}_{d}\right\|+\left\|\boldsymbol{y}_{d}^{(r)}\right\|\right. \\
& \left.+\left\|\boldsymbol{C}_{e}^{\mathrm{T}} \underline{\boldsymbol{e}}\right\|+\|\boldsymbol{F}\|+\frac{1}{2}\left\|\boldsymbol{L} \boldsymbol{R}^{-1} \tilde{\boldsymbol{E}}_{1}\right\|+\left\|\boldsymbol{L} \boldsymbol{C}_{o}^{\mathrm{T}} \boldsymbol{P}_{2} \underline{\hat{\boldsymbol{e}}}\right\|+\frac{1}{2}\left\|\tilde{\boldsymbol{E}}_{1}^{\mathrm{T}}\right\| /\left\|\boldsymbol{L}^{-1}\right\|+\boldsymbol{F}^{U}\right]
\end{aligned}
$$

Where

$I^{*}= \begin{cases}0 & V_{1} \leq \bar{V} \\ 1 & V_{1}>\bar{V}\end{cases}$

$V_{1}=\frac{1}{2} \underline{\tilde{\boldsymbol{e}}}^{\mathrm{T}} \boldsymbol{P}_{1} \underline{\tilde{\boldsymbol{e}}}$

Where $\bar{V}$ is a positive number.

Given certain matrixes $\boldsymbol{Q}_{2}$ and $\boldsymbol{Q}_{3}$, there always exits $\boldsymbol{P}_{2}$ and $\boldsymbol{P}_{3}$, making the following equations.

$\left\{\begin{array}{l}\boldsymbol{A}_{l}^{\mathrm{T}} \boldsymbol{P}_{1}+\boldsymbol{P}_{1} \boldsymbol{A}_{l}-\boldsymbol{P}_{1} \boldsymbol{B}_{l}\left(\boldsymbol{R}^{-1}-\frac{\boldsymbol{I}}{\rho^{2}}\right) \boldsymbol{B}_{l}^{\mathrm{T}} \boldsymbol{P}_{1}+\boldsymbol{Q}_{2}=0 \\ \boldsymbol{P}_{1} \boldsymbol{B}_{l}=\boldsymbol{C}_{l}\end{array}\right.$

$\left(\boldsymbol{A}-\boldsymbol{B} \boldsymbol{C}_{e}^{\mathrm{T}}\right)^{\mathrm{T}} \boldsymbol{P}_{2}+\boldsymbol{P}_{2}\left(\boldsymbol{A}-\boldsymbol{B} \boldsymbol{C}_{e}^{\mathrm{T}}\right)=-\boldsymbol{Q}_{3}$

Where $\rho$ is a designed parameter, $\boldsymbol{R}=\operatorname{diag}\left[\begin{array}{llll}r \boldsymbol{I}_{1} & r \boldsymbol{I}_{2} & \cdots & r \boldsymbol{I}_{n}\end{array}\right], r$ is a positive number, and $\boldsymbol{R}^{-1}-\left(1 / \rho^{2}\right) \boldsymbol{I}>0$.

Applying the control law (11) and the following adaptive law into system (2):

$\dot{\Theta}_{1}=-\gamma_{1} \alpha \boldsymbol{\Phi}_{l}^{\mathrm{T}}(\hat{\boldsymbol{x}}) \tilde{\boldsymbol{E}}_{1}-\gamma_{1} \Theta_{1}$

$\dot{\Theta}_{2 i}=-\gamma_{2} \alpha \boldsymbol{\Phi}_{l}^{\mathrm{T}}(\hat{\boldsymbol{x}}) \tilde{\boldsymbol{E}}_{1} \boldsymbol{u}_{l i}^{\mathrm{T}}-\gamma_{2} \Theta_{2 i}$

$\dot{\Theta}_{3}=-\gamma_{3}(1-\alpha) \boldsymbol{\Phi}_{l}^{\mathrm{T}}(\hat{\boldsymbol{x}}) \tilde{\boldsymbol{E}}_{1}-\gamma_{3} \Theta_{3}$

The overall adaptive scheme guarantees the global stability of the resulting closed-loop system in the sense that all signals involved are uniformly bounded and the tracking error will converge to zero asymptotically. Proof. We choose that:

$$
V=\frac{1}{2} \underline{\underline{\boldsymbol{e}}}^{\mathrm{T}} \boldsymbol{P}_{1} \underline{\tilde{\boldsymbol{e}}}+\frac{1}{2} \underline{\hat{\boldsymbol{e}}}^{\mathrm{T}} \boldsymbol{P}_{2} \underline{\hat{\boldsymbol{e}}}+\frac{1}{2 \gamma_{1}} \tilde{\Theta}_{1}^{\mathrm{T}} \tilde{\Theta}_{1}+\frac{1}{2 \gamma_{2}} \operatorname{tr}\left(\tilde{\Theta}_{2}^{\mathrm{T}} \tilde{\Theta}_{2}\right)+\frac{1}{2 \gamma_{3}} \tilde{\Theta}_{3}^{\mathrm{T}} \tilde{\Theta}_{3}
$$

Then 


$$
\begin{aligned}
\dot{V} & \leq \frac{1}{2} \tilde{\boldsymbol{e}}^{\mathrm{T}}\left[\boldsymbol{A}_{l}^{\mathrm{T}} \boldsymbol{P}_{1}+\boldsymbol{P}_{1} \boldsymbol{A}_{l}\right] \underline{\tilde{\tilde{e}}}+\tilde{\boldsymbol{E}}_{1}^{\mathrm{T}}\left[\varpi-\sigma_{0}\left(\boldsymbol{u}_{b l}+\boldsymbol{u}_{r l}+\boldsymbol{u}_{s l}\right)\right]-\frac{1}{2} \underline{\hat{\boldsymbol{e}}}^{\mathrm{T}} \boldsymbol{Q}_{3} \underline{\hat{\boldsymbol{e}}}+\underline{\hat{\boldsymbol{e}}}^{\mathrm{T}} \boldsymbol{P}_{2} \boldsymbol{C}_{o} \boldsymbol{C}_{e}^{\mathrm{T}} \underline{\tilde{\boldsymbol{e}}} \\
& =\frac{1}{2} \underline{\tilde{\boldsymbol{e}}}^{\mathrm{T} \mathrm{T}}\left[\boldsymbol{A}_{l}^{\mathrm{T}} \boldsymbol{P}_{1}+\boldsymbol{P}_{1} \boldsymbol{A}_{l}\right] \underline{\tilde{\boldsymbol{e}}}-\frac{1}{2} \underline{\tilde{\boldsymbol{e}}}^{\mathrm{T}} \boldsymbol{P}_{1} \boldsymbol{B}_{l} \boldsymbol{R}^{-1} \boldsymbol{B}_{l}^{\mathrm{T}} \boldsymbol{P}_{1} \underline{\tilde{\boldsymbol{e}}}+\underline{\tilde{\boldsymbol{e}}}^{\mathrm{T}} \boldsymbol{P}_{1} \boldsymbol{B}_{l} \varpi-\frac{1}{2} \underline{\hat{\boldsymbol{e}}}^{\mathrm{T}} \boldsymbol{Q}_{3} \underline{\hat{\boldsymbol{e}}} \\
& =\frac{1}{2} \underline{\tilde{\boldsymbol{e}}}^{\mathrm{T} \mathrm{Q}} \boldsymbol{Q}_{2} \underline{\tilde{\boldsymbol{e}}}-\frac{1}{2} \underline{\hat{\boldsymbol{e}}}^{\mathrm{T}} \boldsymbol{Q}_{3} \underline{\hat{\boldsymbol{e}}}+\frac{1}{2} \rho^{2} \varpi^{\mathrm{T}} \varpi-\frac{1}{2}\left(\frac{1}{\rho^{2}} \boldsymbol{B}_{l}^{\mathrm{T}} \boldsymbol{P}_{1} \underline{\tilde{\boldsymbol{e}}}-\rho \varpi\right)^{\mathrm{T}}\left(\frac{1}{\rho^{2}} \boldsymbol{B}_{l}^{\mathrm{T}} \boldsymbol{P}_{1} \underline{\tilde{\boldsymbol{e}}}-\rho \varpi\right)
\end{aligned}
$$

and it can be further written as:

$\dot{V} \leq-\frac{1}{2} \boldsymbol{E}^{\mathrm{T}} \boldsymbol{Q} \boldsymbol{E}+\frac{1}{2} \rho^{2} \varpi^{\mathrm{T}} \varpi$

Because $\varpi \in L_{2}$, then $\boldsymbol{x}, \hat{\boldsymbol{x}}, \underline{\boldsymbol{e}}, \underline{\hat{\boldsymbol{e}}} \in L_{\infty}$, and $\lim _{t \rightarrow \infty} \boldsymbol{E}=\boldsymbol{0}$. We get $\lim _{t \rightarrow \infty} \underline{\tilde{\boldsymbol{e}}}=\boldsymbol{0}$ and $\lim _{t \rightarrow \infty} \underline{\hat{\boldsymbol{e}}}=\boldsymbol{0}$, 又 because $\underline{\boldsymbol{e}}=\underline{\hat{\boldsymbol{e}}}+\underline{\tilde{\boldsymbol{e}}}$ and $\boldsymbol{E}_{1}=\boldsymbol{C}_{e}^{\mathrm{T}} \underline{\boldsymbol{e}}$, so $\lim _{t \rightarrow \infty} \boldsymbol{E}_{1}=\boldsymbol{0}$.

\section{Simulation}

The robotic manipulators' dynamics can be rewritten as[7]:

$\boldsymbol{M}(\boldsymbol{q}) \ddot{\boldsymbol{q}}+\boldsymbol{C}(\boldsymbol{q}, \dot{\boldsymbol{q}}) \dot{\boldsymbol{q}}+\boldsymbol{G}(\boldsymbol{q})+\boldsymbol{F}(\dot{\boldsymbol{q}})+\boldsymbol{\tau}_{\mathrm{d}}=\boldsymbol{\tau}$

The parameters of a two-link robot are:

$$
\begin{aligned}
& \boldsymbol{M}(\boldsymbol{q})=\left[\begin{array}{cc}
p_{1}+p_{2}+2 p_{3} \cos q_{2} & p_{2}+p_{3} \cos q_{2} \\
p_{2}+p_{3} \cos q_{2} & p_{2}
\end{array}\right] \quad \boldsymbol{C}(\boldsymbol{q}, \dot{\boldsymbol{q}})=\left[\begin{array}{cc}
-p_{3} \dot{q}_{2} \sin q_{2} & -p_{3}\left(\dot{q}_{1}+\dot{q}_{2}\right) \sin q_{2} \\
p_{3} \dot{q}_{1} \sin q_{2} & 0
\end{array}\right] \\
& \boldsymbol{G}(\boldsymbol{q})=\left[\begin{array}{c}
p_{4} g \cos q_{1}+p_{5} g \cos \left(q_{1}+q_{2}\right) \\
p_{5} g \cos \left(q_{1}+q_{2}\right)
\end{array}\right] \boldsymbol{F}(\dot{\boldsymbol{q}})=0.02 \operatorname{sgn}(\dot{\boldsymbol{q}}) \\
& \boldsymbol{\tau}_{\mathrm{d}}=d_{1}+d_{2}\|\boldsymbol{e}\|+d_{3}\|\dot{\boldsymbol{e}}\|
\end{aligned}
$$

The simulation values are as follows: $\boldsymbol{p}=\left[\begin{array}{lllll}2.90 & 0.76 & 0.87 & 3.04 & 0.87\end{array}\right]^{\mathrm{T}}, \boldsymbol{d}=\left[\begin{array}{lll}0.1 & 0.2 & 0.6\end{array}\right]^{\mathrm{T}}, g=9.8$, $\Delta \boldsymbol{M}(\boldsymbol{q})=0.2 * \boldsymbol{M}_{0}(\boldsymbol{q}), \quad \Delta \boldsymbol{C}(\boldsymbol{q}, \dot{\boldsymbol{q}})=0.2 * \boldsymbol{C}_{0}(\boldsymbol{q}, \dot{\boldsymbol{q}}), \quad \Delta \boldsymbol{G}(\boldsymbol{q})=0.2 * \boldsymbol{G}_{0}(\boldsymbol{q}) \quad, \quad \boldsymbol{q}_{0}=\left[\begin{array}{ll}1.0 & 1.0\end{array}\right]^{\mathrm{T}} \quad, \quad \dot{\boldsymbol{q}}_{0}=\left[\begin{array}{ll}0.4 & 0.4\end{array}\right]^{\mathrm{T}}$, $\boldsymbol{q}_{\mathrm{d}}=[1+0.2 \sin (\pi t) \quad 1+0.2 * \cos (\pi t)]^{\mathrm{T}}$. And select prober parameters, and we get the simulation result showed in Fig. 1.
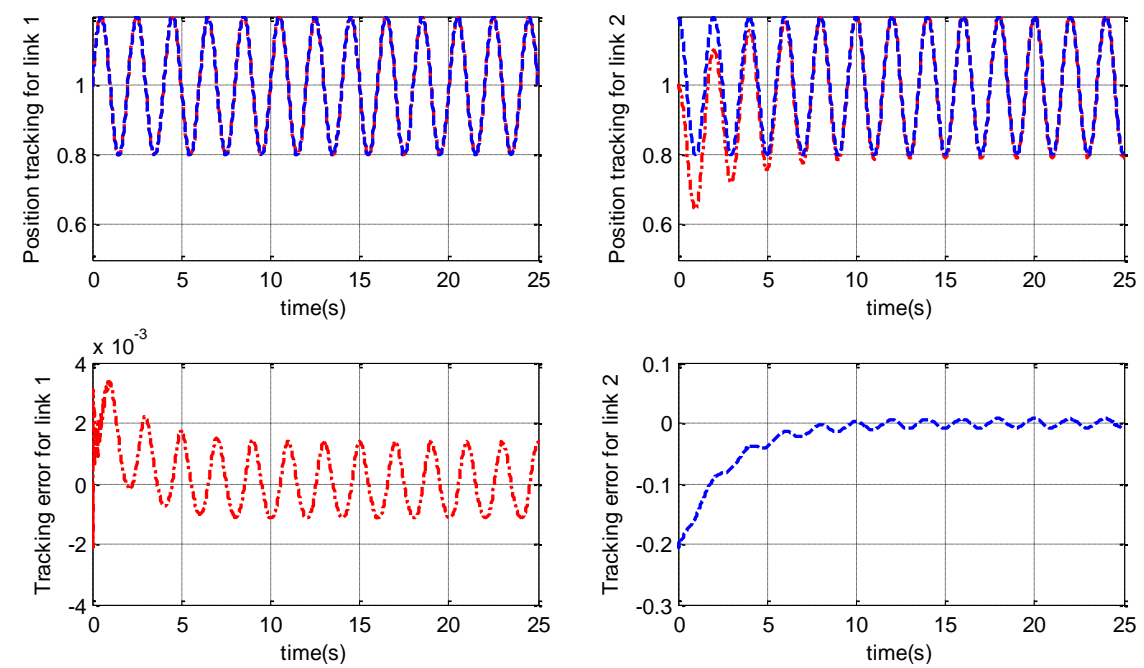

Fig. 1 Simulation Result

\section{Conclusions}

In this study, an adaptive sliding-mode control algorithm is proposed to handle uncertain robotic dynamical systems. To prevent the system state variables unpredictable, this paper also designs an observer to estimate 
the unpredictable states. The control structure and learning rules are derived from a Lyapunov theory extension that guarantee both tracking errors and parameter estimate errors in the closed-loop system are bounded. From the simulation result, it is obvious that the proposed algorithm can achieve good results.

\section{References}

[1] J. Wang, A. B. Rad, and P. Chan, "Indirect adaptive fuzzy sliding mode control: Part I: fuzzy switching," Fuzzy sets and Systems, vol. 122, no. 1, pp. 21-30, 2001.

[2] A. Jalali et al., "Model-free adaptive fuzzy sliding mode controller optimized by particle swarm for robot manipulator," International Journal of Information Engineering and Electronic Business, vol. 5, no. 1, pp. 68, 2013.

[3] J. Jantzen, Foundations of fuzzy control: a practical approach: John Wiley \& Sons, 2013.

[4] F. Piltan et al., 'Design sliding mode controller of with parallel fuzzy inference system compensator to control of robot manipulator," IAES International Journal of Robotics and Automation vol. 2, no. 4, pp. 149-162, 2013.

[5] Y.-K. Yang et al., "A novel self-constructing radial basis function neural-fuzzy system," Applied Soft Computing, vol. 13, no. 5, pp. 2390-2404, 2013.

[6] Y. Li et al., "Adaptive fuzzy control of uncertain stochastic nonlinear systems with unknown dead zone using small-gain approach,” Fuzzy Sets and Systems, vol. 235, pp. 1-24, 2014.

[7] K. Lochan, and B. Roy, "Control of two-link 2-DOF robot manipulator using fuzzy logic techniques: a review." pp. 499-511.

[8] Y. Li et al., "Adaptive fuzzy robust output feedback control of nonlinear systems with unknown dead zones based on a small-gain approach," Fuzzy Systems, IEEE Transactions on, vol. 22, no. 1, pp. 164-176, 2014.

[9] R.-J. Lian, “Adaptive self-organizing fuzzy sliding-mode radial basis-function neural-network controller for robotic systems,” Industrial Electronics, IEEE Transactions on, vol. 61, no. 3, pp. 1493-1503, 2014.

[10]F. Piltan et al., "Design robust fuzzy sliding mode control technique for robot manipulator systems with modeling uncertainties," International Journal of Information Technology and Computer Science vol. 5, no. 8, pp. 123, 2013. 